\title{
ANÁLISIS DISCRIMINANTE APLICADO A MODELOS DE PREDICCIÓN DE QUIEBRA
}

\author{
DISCRIMINANT ANALYSIS APPLIED TO PREDICTION MODELS IN BANKRUPTCY
}

Janet Cecibel Aldazábal Contreras *

Alberto Fernando Napán Vera**

Docentes Auxiliares de la Facultad de Ciencias Contables

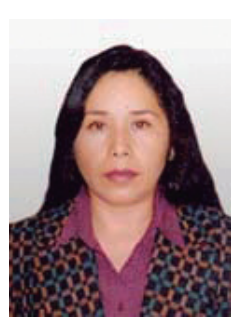

Universidad Nacional Mayor de San Marcos-UNMSM / Lima-Perú

[Recepción: Setiembre de 2014/ Conformidad: Octubre 2014]

\section{RESUMEN}

Las empresas interactúan cada vez más con clientes y proveedores. Esta situación hace necesaria una adecuada gestión del riesgo para prevenir situaciones de insolvencia financiera. Una empresa es técnicamente insolvente cuando no tiene efectivo suficiente para efectuar sus pagos inmediatos. Por lo tanto, uno de los puntos a estudiar es la solvencia financiera y el riesgo a la quiebra de su cartera de clientes. Para este fin, existen técnicas que permiten medir esta posibilidad de insolvencia de una empresa. Entre las más confiables está el modelo $\mathrm{Z}$ de Altman, el cual está basado en la técnica estadística del Análisis Discriminante Múltiple. Este modelo emplea ratios financieros para determinar el riesgo financiero y predecir si una empresa es saludable desde el punto de vista financiero o se encuentra en camino a ser insolvente. Se presentan los resultados hallados por Altman y la revisión del modelo original planteado por él.

\section{Palabras clave:}

Quiebra; modelos de predicción; ratio; Análisis Discriminante Múltiple; Altman.

\begin{abstract}
The companies increasingly interact with clients and providers. This situation requires adequate risk management to prevent situations of financial distress. A company is technically insolvent when it has enough cash to make immediate payments. Therefore, one of the points to study is the financial solvency and risk to the bankruptcy of its customer base. To this end, there are techniques to measure the possibility of insolvency of a company. The most reliable is the Altman $\mathrm{Z}$ model, which is based on the statistical technique of Multiple Discriminant Analysis. This model uses financial ratios to determine the financial risk and predict whether a company is healthy from a financial point of view, or is on its way to become insolvent. The results found by Altman and revision of the original model proposed by him is presented.
\end{abstract}

\section{Keywords:}

Bankruptcy; prediction models; ratio; Multiple Discriminant Analysis; Altman.

\footnotetext{
* Lic. Investigación Operativa, Colegiado en el COMAP. E-mail:jaldazabalc@unmsm.edu.pe
}

** Lic. Investigación Operativa, Colegiado en el COMAP. E-mail:anapanv@unmsm.edu.pe 


\section{INTRODUCCIÓN}

En la actualidad es importante contar con herramientas que permitan medir el riesgo financiero de una empresa, más aun en un mercado cada vez más amplio y globalizado donde se interactúa con clientes y proveedores. Es necesario entonces, para cualquier empresa realizar un seguimiento de la exposición al riesgo de la cartera de clientes actuales y potenciales. Una adecuada gestión del riesgo permitirá prevenir situaciones de insolvencia financiera.

Uno de los modelos más conocidos y confiables para medir la insolvencia de una empresa es el modelo $Z$ de Altman, el cual está basado en la técnica estadística del Análisis Discriminante Múltiple.

El objetivo de este trabajo es describir los antecedentes y la aplicación de este modelo.

\section{QUIEBRA DE UNA EMPRESA}

Ante la pregunta de cuáles son los factores que conduce a la quiebra a una empresa, algunas corrientes de pensamiento acusan a problemas de liquidez, deficiente administración, a los ciclos económicos y cambios en el mercado que favorecen a ciertas empresas, mientras que otras se vuelven ineficientes en ese entorno.

Cabe preguntarse si existe entonces alguna técnica para determinar si una empresa tiende o está destinada a caer en crisis.

No podemos determinar sin posibilidad de error si una empresa quebrará o no, pero sí podemos estimar la probabilidad de quiebra. Para este fin se han desarrollado múltiples modelos matemáticos.

El diccionario de la RAE define la quiebra como: "Juicio por el que se incapacita patrimonialmente a alguien por su situación de insolvencia y se procede a ejecutar todos sus bienes en favor de la totalidad de sus acreedores".

Una empresa es técnicamente insolvente cuando no tiene efectivo suficiente para efectuar sus pagos inmediatos.

Por lo tanto, el punto de partida para el análisis de quiebra es la información contable.
En este sentido, es útil entonces estudiar los modelos de predicción de quiebra existentes que permitan predecir la futura solvencia de una empresa.

Los modelos de predicción de quiebra intentan estimar cuáles son los factores que determinan este escenario financiero.

\section{ANÁLISIS DISCRIMINANTE}

El análisis multivariante es un conjunto de métodos estadísticos y matemáticos destinados a describir e interpretar los datos que provienen de la observación de varias variables estadísticas, estudiadas conjuntamente.

Asumiendo que un conjunto de casos de estudio está ya clasificado en una serie de grupos; es decir, se sabe previamente a qué grupos, pertenecen, el análisis discriminante es una técnica del análisis Multivariante cuyo objetivo es encontrar la combinación lineal de las variables independientes que mejor permita diferenciar (discriminar) a los grupos. Una vez encontrada esa combinación (la función discriminante) podrá ser usada para clasificar nuevos casos de estudio. Aprovecha las relaciones existentes entre una gran cantidad de variables independientes para maximizar la capacidad de discriminación.

$\mathrm{El}$ análisis discriminante entonces permite estudiar las diferencias entre dos (en el caso del análisis simple) o más (estaríamos ante el análisis discriminante múltiple) grupos definidos a priori, con respecto a varias variables simultáneamente.

Un estudio es eficiente si logra diferenciar correctamente empresas que están en situación de insolvencia de empresas que no lo están.

Estadísticamente podemos identificar dos tipos de errores:

Error tipo I: Se clasifica como empresa no insolvente a una que está en quiebra.

Error tipo II: Se clasifica como empresa insolvente a una que no está en quiebra.

Un modelo se considera bueno si minimiza el número de errores.

En la práctica, no es posible reducir los dos tipos de errores al mismo tiempo, pero se busca un punto 
de equilibrio. Por ejemplo, este equilibrio lo podemos encontrar en la política de créditos de un banco. Una política de otorgamiento de créditos conservadora intenta reducir el error tipo II. El costo de esta política es la no obtención del interés del préstamo no concedido a las empresas con una buena situación financiera que; sin embargo, fueron clasificadas como insolventes.

Beaver, en 1966, utilizando el análisis discriminante simple planteó un modelo de predicción de quiebra. Altman, en 1968, utilizando el análisis discriminante múltiple planteó también un nuevo modelo de predicción de quiebra, el cual ha sido ampliamente difundido.

\section{USO DE RATIOS FINANCIEROS EN EL ANÁLISIS DE QUIEBRA}

Las acciones realizadas por las empresas están representadas en forma de transacciones que son registradas por la contabilidad para generar estados financieros, que son la principal herramienta de análisis de una empresa. Con las cuentas de los estados financieros se pueden establecer un conjunto de razones o ratios que son útiles para determinar la situación de la empresa.

Los modelos de análisis discriminante múltiple emplean ratios financieros como variables independientes. El motivo es que permiten facilitar la comparación entre empresas independientemente de su tamaño.

Debemos considerar que los ratios financieros de un sector productivo de la economía en particular pueden demandar análisis de un ratio específico. Los ratios no necesariamente son comparables entre empresas de distintos rubros.

Tradicionalmente, siempre se ha debatido sobre qué ratios debemos priorizar para predecir el fracaso de una empresa. Beaver considera que el fracaso de una empresa se define como la incapacidad de ésta para cumplir con sus deudas. Entonces, es lógico asignarle mayor importancia a las magnitudes relacionadas con la obtención de flujos de caja para medir una situación de insolvencia.

Luego de esta primera selección de ratios a incluir en el modelo, se establece un nuevo proceso que tiene como objetivo reducir el número de variables del modelo final.

En la gran mayoría de los modelos de predicción del fracaso de un empresa, los ratios de rentabilidad, seguidos de los ratios de liquidez, son las variables más significativas para determinar la futura situación de insolvencia de una empresa.

\section{USO DEL ANÁLISIS DISCRIMINANTE MÚLTIPLE (ADM)}

El uso del ADM fue propuesto por Altman como modelo predictivo de quiebra de una empresa.

El modelo de ADM combina la información de diversas variables financieras en un análisis interdependiente.

El primer paso es determinar los grupos de clasificación, en nuestro caso:

a) Insolvencia

b) Solvencia

Esta predicción de pertenencia o no a un grupo se constituye en la variable dependiente.

Establecidos los grupos, se recolectan los datos de las empresas que los componen. El modelo de $\mathrm{ADM}$ permite determinar un conjunto con los coeficientes que permitan discriminar mejor a los grupos de estudio. Estos coeficientes son ponderados y se construye una ecuación lineal con el cual clasificar a la empresa en estudio.

En 1968 Edward Altman, catedrático de New York University, desarrolló el modelo diseñado para suministrar una evaluación mejorada de la solvencia de clientes y proveedores, y obtener decisiones de inversión más seguras.

Desde ese momento, el modelo de Altman-Z se ha convertido en un método popular para analizar la salud de una empresa y determinar la probabilidad de su quiebra dentro de uno a dos años.

Una de las ventajas del modelo de ADM planteado por Altman es la reducción del número de variables dependientes, en nuestro caso específico a solo una: predicción de insolvencia.

La función discriminante tiene la forma:

$$
\mathrm{Z}=\mathrm{V}_{1} \mathrm{X}_{1}+\mathrm{V}_{2} \mathrm{X}_{2}+\ldots+\mathrm{V}_{\mathrm{n}} \mathrm{X}_{\mathrm{n}}
$$


La fórmula estaba originalmente basada en los datos de 66 empresas manufactureras que cotizaron en bolsa. La mitad de estas se habían declarado en quiebra dentro de los dos años previos, y la mitad era empresas en marcha. Las 66 empresas tenían activos por más de $\$ 1$ millón.

La estimación obtenida por el modelo Z-Altman no es $100 \%$ segura. Sin embargo, ha probado ser uno de los mejores modelos estadísticos para determinar el riesgo de quiebra y la salud de una empresa. En general, un valor de $\mathrm{Z}$ bajo indica problemas, aunque como hemos señalado esto no es determinante para el futuro de la empresa.

Altman calculó 22 ratios financieros clasificados en 5 categorías estándar: liquidez, rentabilidad, apalancamiento, solvencia y actividad. Después de numerosas corridas, se seleccionaron las 5 variables que juntas dieron el mejor resultado en la predicción de la insolvencia.

El cálculo del valor de $\mathrm{Z}$ está basado completamente sobre las cifras de los reportes financieros de una empresa. Utiliza 7 cifras tomadas del balance y el Estado de Resultados. Cinco ratios son extrapolados de estos siete datos.

Según los valores que obtuviera $\mathrm{Z}$ al ser evaluado con las cifras de la empresa estudiada, el resultado puede ser:

a) Saludable: la empresa no tendrá problemas de insolvencia en el futuro;

b) Zona gris: la empresa tiene altas posibilidades de caer en insolvencia dentro de los próximos 2 años, sugiere reconsiderar estrategias e implementar cambios.

c) Enferma: la probabilidad de insolvencia en el futuro es muy alta, podría presentar quiebra en 2 años.

Para empresas manufactureras que cotizan en bolsa, la función discriminante final fue:

$$
\mathrm{Z}=1.23 \mathrm{X}_{1}+1.4 \mathrm{X}_{2}+3.3 \mathrm{X}_{3}+0.6 \mathrm{X}_{4}+0.99 \mathrm{X}_{5}
$$

Donde:

$\mathrm{X}_{1}=$ Capital de trabajo/Activo total.

$\mathrm{X}_{2}=$ Utilidades retenidas/Activo total.

$\mathrm{X}_{3}=$ Utilidades antes de intereses e impuestos/Activo total.
$\mathrm{X}_{4}=$ Valor de mercado de las acciones/Pasivo total.

$\mathrm{X}_{\mathrm{s}}=$ Ventas/Activo total.

Cada ratio tiene una razón para ser parte del modelo.

$\mathrm{X}_{1}$ : En el análisis financiero, el uso de los activos totales como denominador es habitual, por lo que es lógico que haya ese denominador en cuatro de los cinco ratios. El activo total en el denominador permite comparar los otros elementos del balance o del estado de ganancias y pérdidas. El numerador es la diferencia entre los activos a corto plazo y pasivos a corto plazo. Puede ser negativo si los pasivos a corto plazo son mayores que los activos a corto plazo. Esta relación proporciona información acerca de la situación financiera a corto plazo de la empresa. Mayor nivel de capital de trabajo en comparación con el total de activos significa mayor nivel de liquidez. Altman distinguió a X1 como una de los ratios más importantes.

$\mathrm{X}_{2}$ : Muestra la calidad de la estructura de capital. Si la empresa tiene pérdidas durante varios años consecutivos, este número será negativo. Un factor importante para una empresa es tener ganancias retenidas para mostrar a los inversionistas que hay planes de reinversión y crecimiento. Además, la empresa con un alto nivel de utilidades retenidas puede financiar sus proyectos sin requerir préstamos.

$\mathrm{X}_{3}$ : Es una razón de rentabilidad y es por ello, su elevado peso en el modelo. El numerador es el EBIT (utilidad antes de intereses e impuestos). El propósito de $\mathrm{Z}$ es predecir el éxito o el fracaso y es obvio que las empresas con pérdidas tendrán menor ratio y una mayor posibilidad de ir a la quiebra.

$\mathrm{X}_{4}$ : Es la variable con el peso más bajo y el motivo es su numerador: el valor de mercado de las acciones. Estos datos se obtienen de los mercados de capitales y, eventualmente, puede ser fácil de manipular. El denominador es el pasivo total por lo que la relación muestra si el valor de mercado del patrimonio excede al valor en libros de los pasivos. Existe un riesgo potencial de una empresa si este no es el caso. Entonces la empresa debería encontrar la forma de disminuir sus pasivos. 
$\mathrm{X}_{5}$ : Este ratio muestra la velocidad de la circulación de los activos. Se le ha asignado el mayor peso en el modelo pero como veremos más adelante Altman decidió excluirlo del modelo Z2, ya que consideró que es fácil de manipular los ingresos totales a través de los métodos contables.

El criterio para interpretar $Z$ es:

$\mathrm{Z}>=2.99$ : Saludable;

$2.99<=\mathrm{Z}<=1.81$ : Zona gris;

\section{$\mathrm{Z}<1.81$ : Enferma}

Debido a que este modelo aplicaba sólo a empresas manufactureras que cotizaban en bolsa, Altman hizo una revisión del mismo y obtuvo dos nuevas versiones, el Z1 y el Z2.

Modelo Z1 de Altman (Para compañías manufactureras que no cotizan en bolsa):

En el ratio de $\mathrm{X}_{4}$, se reemplaza el valor de mercado de las acciones con el valor del capital contable.

$\mathrm{Z}=0.717 \mathrm{X}_{1}+0.847 \mathrm{X}_{2}+3.107 \mathrm{X}_{3}+0.42 \mathrm{X}_{4}+0.998 \mathrm{X}_{5}$

Donde:

$\mathrm{Z}>=2.99$ : Saludable;

$2.99<=\mathrm{Z}<=1.23$ : Zona gris;

$\mathrm{Z}<1.23$ : Enferma
Modelo Z2 de Altman (Para todo tipo de empresas): El ratio $\mathrm{X}_{5}$ es excluido del cálculo de puntaje de $\mathrm{Z}$. $\mathrm{Z}=6.56 \mathrm{X}_{1}+$ de $3.26 \mathrm{X}_{2}+6.72 \mathrm{X}_{3}+1.05 \mathrm{X}_{4}$

Donde:

$\mathrm{Z}>=2.6$ : Saludable;

$2.6<=\mathrm{Z}<=1.10$ : Zona gris;

$\mathrm{Z}<1.10$ : Enferma

\section{CASO PRÁCTICO: CAJA MUNICIPAL DE AHORRO Y CRÉDITO PISCO}

La Superintendencia de Banca, Seguros y AFP (SBS), dispuso la liquidación de la Caja Municipal de Ahorro y Crédito de Pisco, el 21 de mayo de 2014 mediante la Resolución SBS No 3028-2014. El motivo fue el incumplimiento de los compromisos asumidos en el Plan de Recuperación Financiera que tenía como propósito de superar la delicada situación financiera por la que atravesaba. A marzo de 2014, la Caja figuraba entre las entidades con mayor morosidad entre las cajas municipales. A su vez, estuvo entre las entidades con el ROE más bajo y sufrió de bajísima liquidez de soles (activos/pasivos promedio mensual, según la SBS).

Cuadro No 01: Situación financiera Caja Pisco

\begin{tabular}{|c|c|c|c|c|}
\hline & 2010 & 2011 & 2012 & 2013 \\
\hline Activo Líquido & 8,342 & 7,329 & 12,169 & 9,956 \\
\hline Pasivo de corto plazo & 23,575 & 35,086 & 43,705 & 40,415 \\
\hline Capital de trabajo & $-15,233$ & $-27,757$ & $-31,536$ & $-30,459$ \\
\hline Activo total & 47,418 & 58,658 & 66,585 & 63,487 \\
\hline Pasivo total & 40,142 & 52,007 & 59,564 & 56,328 \\
\hline Utilidades retenidas & 0 & 146 & -440 & -235 \\
\hline Utilidades antes de int. e imp. & 649 & -586 & 775 & $-2,734$ \\
\hline Capital contable & 7,276 & 6,652 & 7,021 & 7,159 \\
\hline
\end{tabular}

Cuadro No 02: Resultado de las Variables Modelo

\begin{tabular}{|c|c|c|c|c|}
\hline & 2010 & 2011 & 2012 & 2013 \\
\hline $\mathrm{x} 1$ & -0.32 & -0.47 & -0.47 & -0.48 \\
\hline $\mathrm{x} 2$ & 0.00 & 0.00 & -0.01 & 0.00 \\
\hline $\mathrm{x} 3$ & 0.001 & -0.01 & 0.01 & -0.04 \\
\hline $\mathrm{x} 4$ & 0.18 & 0.13 & 0.12 & 0.13 \\
\hline Coeficiente $\mathrm{z}$ & -1.38 & -3.03 & -2.93 & -3.32 \\
\hline
\end{tabular}

Fuente: Superintendencia de Banca, Seguros y AFP. Elaboración propia. 
Gráfico No 01: Resultado del Modelo

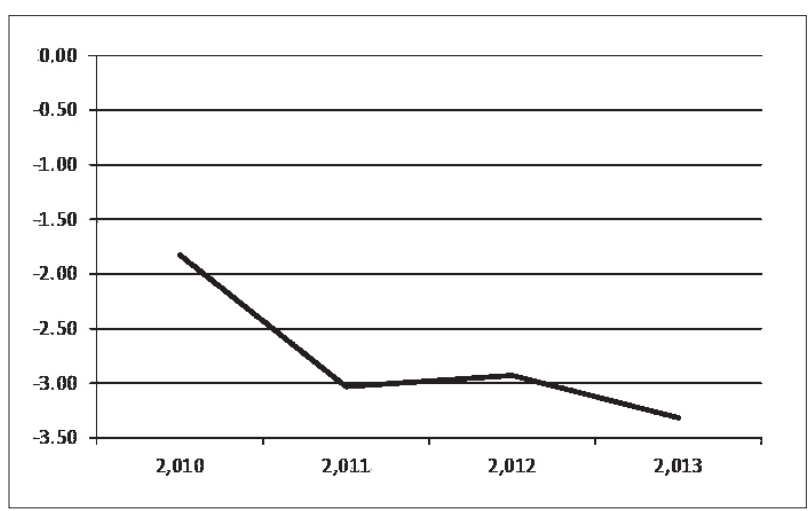

Fuente: Superintendencia de Banca, Seguros y AFP. Elaboración propia.

Observamos un coeficiente muy negativo. La explicación es el tipo de negocio.

La variable $\mathrm{X}_{1}$, que involucra al capital de trabajo, posee el coeficiente más alto, y esto se justifica por ejemplo, para las industrias manufactureras, donde el tamaño del inventario y los plazos de las cuentas por cobrar son importantes para determinar los niveles del ratio.

El capital de trabajo es el activo corriente menos el pasivo corriente. Para los fabricantes, los componentes del activo corriente son dinero en efectivo, valores negociables, cuentas por cobrar e inventarios. Los pasivos corrientes son las deudas por un plazo de 1 año a partir de la fecha del balance. Estas deudas se pagan con los activos corrientes.

La capacidad de una empresa para cumplir sus obligaciones, para crecer y aprovechar las oportunidades es frecuentemente determinada por su capital de trabajo.

En el ciclo de la producción manufacturera, los inventarios en almacén a la espera de ser vendidos son sólo efectivo potencial. Lo esencial del ratio $\mathrm{X}_{1}$ es medir la convertibilidad de activos en efectivo para casos de emergencia. Para calcular este ratio, es necesario deducir los inventarios de productos del activo corriente.

Una empresa con constante déficits de explotación erosiona los activos corrientes en comparación con el total de activos.

A diferencia de las empresas manufactureras, los bancos no tienen inventario de productos de proce- so o inventario productos terminados. Además, la mayor parte de los pasivos están compuesto por las obligaciones con sus clientes en forma de depósitos.

Para reflejar las operaciones relacionadas a la actividad bancaria habría que considerar los componentes equivalentes para los activos corrientes como caja, banco y corresponsales, y préstamos a corto plazo.

Como se mencionó el modelo Z-Altman no es un indicador perfecto. Por ejemplo:

- No considera quiebras causadas por los factores aparte de aquellos que aparecen sobre el balance.

- Se verá afectada por empresas que manipulan su información contable para conservar su imagen corporativa.

- No es útil para las nuevas compañías con poco o ningún ingreso.

- No es adecuado para evaluar empresas pequeñas.

\section{CONCLUSIONES}

1. El modelo no predice cuando una empresa se declarará en quiebra. Lo que hace es medir el desempeño financiero y proporcionar criterios que pueden sugerir la ocurrencia de ello.

2. Debemos considerar además que, Altman utilizó la información financiera de un número relativamente pequeño de empresas en un escenario de hace más de 45 años para construir el modelo. Es también importante recordar que sigue vigente hasta hoy.

3. Sin embargo, hasta ahora, el modelo Z-Altman ha probado ser una herramienta importante para evaluar la salud de una empresa, la posibilidad de quiebra, y la solvencia en su conjunto.

4. Este modelo, al ser uno de los más aplicados y difundidos, ha sido revisado y adecuado a las condiciones y normatividad de distintos países.

5. Esta herramienta no debe ser tomada como una prueba absoluta de solvencia de una empresa, pero debe ser considerada como una herramienta importante para su evaluación.

6. Para empresas del sector financiero, es necesario una modificación del modelo que refleje la real actividad que se desea medir. 


\section{REFERENCIAS BIBLIOGRÁFICAS}

1. ALTMAN, E. I.(1968) "Financial ratios, discriminate analysis and the prediction of corporate bankruptcy," Journal of Finance, September 1968, pp. 589-609.

2. BEAVER, W. (1968). Financial ratios as predictors of failure. Journal of Accounting. Research, 3, 71-102.

3. BOLTEN, S. (1981). Administración Financiera. 1era. Ed. Edit. Limusa S.A. de C.V., México, D.F., México.

4. CALANDRO, J.JJr. (2007). Considering the utility of altman's Z-score as a strategic assessment and performance management tool. Strategy \& Leadership, 35(5), 37. doi:http://dx.doi. org/10.1108/10878570710819206
5. CUADRAS, C. M. (2014).Nuevos Métodos de Análisis Multivariante. 1era. Ed. Edit. CMC Editions Barcelona, España.

6. NARAYANAN, L. (2010). How to calculate altman Z score of customers and suppliers. IOMA's Report on Managing Credit, Receivables \& Collections, 10(3), 12-14. Retrieved from http:// search.proquest.com/docview/200112554?accountid $=12268$

7. MORA, A. (1995) Utilidad de los Modelos de Predicción de la Crisis Empresarial. Revista Española de Financiación y Contabilidad. Vol. 24 N83:281-300.

8. http://www.sbs.gob.pe/ (visitado el 08-092014) 